\title{
Percepciones de estudiantes de Pedagogía en Matemática acerca de sus competencias TIC: un estudio de caso*
}

\author{
Mauricio E. Moya Márquez $z^{\star \star}$
}

\section{RESUMEN}

Este artículo se centra en los resultados de un estudio cuyo propósito fue develar las percepciones de futuros profesores de matemáticas e informática educativa de la UCSH, acerca de sus competencias en el uso de tecnologías digitales en la escuela. El estudio fue realizado en el contexto de una línea internacional de investigación sobre el uso de las TIC en la enseñanza y el aprendizaje en una sociedad digital. La metodología incluyó un cuestionario y entrevistas. A los participantes se les preguntó cómo percibían sus conocimientos y competencias en el uso de la tecnología digital como recurso para promover el aprendizaje de las matemáticas. Los resultados más importantes mostraron que ellos se percibían mejor preparados en el "conocimiento sobre estas tecnologías" y en los "aspectos técnicos", que en la pedagogía y gestión involucrados en el uso de estos recursos en la escuela. Además, los participantes reportaron una baja participación en proyectos innovadores destinados a incorporar estas tecnologías en la enseñanza y el aprendizaje.

Palabras clave: Competencias digitales docentes, tecnologías de la información y comunicación, informática educativa, formación docente, educación matemática.

\section{Perceptions by Mathematics Pedagogy students about their ICT skills: A case study}

\section{ABSTRACT}

This article focuses on the results of a study aimed to unveiling perceptions that future Teachers of Mathematics and Computers in Education of UCSH have about their skills in the use of digital technologies at School. The study was done in the context of an international line of research on the use of ICT for teaching and learning in a digital society. The methodology included a questionnaire and interviews. Participants in the study were asked how they perceived their knowledge and skills in the use of digital technology as a resource to promote mathematics learning. The most important results showed that participants perceived themselves better prepared in the "knowledge about these technologies" and, in the "technical aspects of these technologies" than in the

Artículo basado en los resultados del trabajo de Tesis para optar al grado de Máster en "Entornos de enseñanza y aprendizaje mediados por tecnologías digitales", a través de un programa especial de la UCSH con la Universidad de Barcelona. Finalizado en septiembre de 2016. https://www.ub.edu/portal/web/educacion/masteres-universitarios/-/ensenyament/ detallEnsenyament/1060454

* Profesor de Estado en Matemática y Computación. Magíster en Educación, Universidad de Santiago de Chile. Docente Universidad Católica Silva Henríquez, Chile. E-mail: mmoyam@ucsh.cl 
pedagogy and management involved in the use of these resources at School. Additionally, respondents reported a low participation in innovative projects intended to incorporate these technologies in teaching and learning at School.

Keywords: Teacher Digital Competences, Information and Communication Technologies, Educational Informatics, Teacher Training, Mathematics Education.

\section{Introducción}

En la actualidad el acceso al conocimiento ha dejado de ser lento, escaso y estable (Torres y Arras, 201 I). Marc Prensky agrega que los "nativos digitales" no conocen el concepto de lento en esta época (Enlaces, 2009). Por ello las nuevas modalidades de enseñanza apoyadas con TIC se están consolidando en varias partes del mundo. En pocas décadas la tecnología computacional ha jugado un importante rol en el acceso a la información, el esparcimiento de la creatividad, el fomento de la colaboración y el trabajo en todo el mundo (Gates, 2002, citado en Matus, 2013).

Estamos en presencia de una nueva era, la era del conocimiento y donde se transforma paulatinamente la forma de transmisión oral y escrita a una forma de transmisión digital (UNESCO, 2005). Los nativos digitales han pasado su vida acompañados de la tecnología a través de los PC, videojuegos, reproductores de música, cámaras digitales y teléfonos móviles. Por el contrario, los "inmigrantes digitales", los adultos, los padres, hemos tenido que aprender a usar esta tecnología digital (Prensky, 200I). En otros contextos, tal vez más apropiado, se habla de los estudiantes como New Millenial Learners (Pedró, 2006).

En UNESCO (2005) ya se plantea un enfoque que implica el fomento de las competencias TIC en los centros educativos. La discusión se centra en la importancia de contar con habilidades TIC para el desarrollo personal y social. Por cierto, esto afecta a todos los países incluyendo los miembros de la OCDE (Aypay, 2010, citado en Torres y Arras, 201I). Claramente constituye un factor estratégico que contribuye al crecimiento con equidad en países de la región. En respuesta a esta tendencia, los gobiernos, a través de sus políticas educacionales, han apostado a la innovación, incrementando la inversión en computadores y conectividad a través de los años, 
ofreciendo a las instituciones educativas mejores herramientas (Matus, 2013).

Con la información del primer Censo TIC realizado en 2012, en nuestro país se ha llegado a obtener una tasa de 6 alumnos por computador en colegios municipales, I4 en colegios particulares subvencionados y II en los particulares pagados. En cuanto a conectividad, este Censo reveló un 66\% para los municipales, 80\% en los colegios particulares subvencionados y $95 \%$ de los particulares pagados. En términos concretos, se ha disminuido la brecha digital en el acceso a la tecnología y las comunicaciones en beneficio de favorecer el aprendizaje de los estudiantes (Enlaces, 2013).

Sin embargo, la sola disponibilidad de tecnología no asegura que los profesores en ejercicio y los futuros profesores estén listos para usar estas herramientas tecnológicas en el aprendizaje de la matemática y las ciencias (Matus, 2013). Aun cuando los docentes utilizan cada vez más los PC para sus labores pedagógicas, solo un 40\% del tiempo los usarían para labores de enseñanza y no más de IO horas semanales, según el censo TIC 2012, sabiéndose muy poco acerca de qué actividades realmente se realizan en ese tiempo semanal y si ellas están relacionadas con el currículo escolar (Enlaces, 2013).

Nuevas generaciones de profesores están formándose en diferentes instituciones educativas. Ellos están interactuando con la tecnología y por cierto muchas esperanzas se siembran al respecto. Un estudio exploratorio realizado por Lei (2009) con estudiantes de pedagogía de una universidad del noreste de los EE.UU. reveló que los estudiantes tenían una visión muy positiva acerca de la tecnología, pero más bien moderada acerca de su propia capacidad y actitud para usarla en sus futuras clases (Matus, 20I3).

Según Lei, los estudiantes para profesores están muy bien capacitados en el uso de tecnología básica pero no están muy familiarizados con tecnologías más avanzadas, el uso de aplicaciones web 2.0 está más bien limitado a las aplicaciones de las redes sociales, pero carecen de un conocimiento profundo y experiencias acerca aplicaciones web 2.0 que sí tienen grandes aplicaciones en clases (Matus, 2013). Además, los futuros profesores no tienen experiencias ni 
conocimientos previos en usar tecnologías especiales para las clases, como dispositivos móviles, testers, probes, pizarras digitales, etc.

Según el NCTM (2000), la tecnología puede aumentar los niveles de motivación, acercando las materias de estudio a su mundo real. Por ejemplo, en educación matemática, computadores son sugeridos como importantes herramientas para el aprendizaje y la enseñanza más efectiva. Más aún, se ha visto que, en el contexto de un buen programa de matemáticas, el uso de tecnología incrementa el rango de contenidos y problemas que los estudiantes pueden trabajar o resolver (Masalsky \& Elliot, 2005, citados por Matus, 2013).

\section{Antecedentes y problema de investigación}

Las competencias TIC, o bien las percepciones respecto de las competencias TIC de los estudiantes universitarios y futuros profesores, constituyen líneas de investigación en la literatura. A la luz de los estudios realizados es posible concluir que la percepción de los estudiantes sobre sus competencias TIC varía, dependiendo de los recursos tecnológicos involucrados (Cabero y Llorente, 2006; Arras, Torres y García-Valcárcel, 201 I; Ortiz, Peñaherrera y Ortega, 2012). En otros casos se manifiesta una alta valoración respecto del uso de las TIC en los procesos educativos, o bien los cambios de rol del docente. Gutiérrez, Palacios y Torrego (2010) aportan información interesante respecto de los modelos educativos en la preparación de futuros profesores.

Prendes, Castañeda y Gutiérrez (2010) revelan que las carencias mayores en los futuros docentes tienen que ver con habilidades de colaboración, liderazgo y gestión de información en grupos. Hay estudios que no solo abordan la competencia digital, sino que involucran la actitud hacia las TIC. "Para que el aprendizaje de las Tic sea provechoso y significativo es necesario que las personas tengan una actitud favorable hacia las mismas" (Torres, 20II, p. 7). Estudios más recientes revelan que los futuros docentes “( ...) tienen un nivel aceptable en las habilidades digitales básicas, pero no disponen de un nivel adecuado en la aplicación didáctica de las TIC (Esteve, 20I5, p. 27). 
Estudios nacionales desarrollados en la Universidad Católica Silva Henríquez (UCSH) con estudiantes de pedagogía aportan información relevante sobre el nivel en el uso de las TIC y sus percepciones sobre las prácticas tecnológicas. Por ejemplo, Bahamondes, Aguilar y Torrealba (2010) y Martínez (2015).

Como parte del contexto, en la Escuela de Educación Matemática e Informática Educativa (EEMIE) de la UCSH se está llevando a cabo un rediseño curricular respecto de la carrera de Pedagogía en Matemática e Informática Educativa (PMIE), que además incorpora modificaciones a raíz del último proceso de Acreditación Académica, y que justamente involucra una innovación en el área de Informática Educativa. En el informe de autoevaluación de la carrera, uno de los puntos propuestos a mejorar tiene que ver con la articulación entre la línea disciplinar y la componente de Informática Educativa. Lo cual conecta con una necesidad evidenciada en terreno el $2^{\circ}$ semestre de 20I5. De los I5 proyectos de tesis presentados por los estudiantes de seminario, solo en dos casos hubo una conexión moderada con el tema de las TIC. Por otra parte, los alumnos en práctica profesional utilizan en general poco las herramientas tecnológicas (supervisión de prácticas).

A partir del problema enunciado y la consideración de los estándares TIC para la formación inicial docente, la pregunta que guió la presente investigación fue la siguiente: ¿cuáles son las percepciones de los estudiantes del último año y egresados de la carrera de PMIE en 2015 acerca de sus competencias TIC, las herramientas fundamentales para su labor docente y un modelo pedagógico para la enseñanza con TIC?

\section{Marco teórico}

El concepto de "competencia" es complejo y en el mundo profesional se ha convertido en sinónimo de idoneidad, suficiencia, capacidad, habilidad, maestría o excelencia (MINEDUC, 2007).

Perrenoud (2008) insiste sobre la necesidad de focalizar la enseñanza más en la formación de competencias y menos en la memorización de contenidos o en la adquisición de aprendizajes sin contexto. Pero 
esa formación por competencias no siempre es entendida como una meta o fin de la enseñanza, siendo este concepto un objetivo nuevo en la formación de los estudiantes.

Le Boterf conceptualiza la competencia como "el saber actuar en un contexto de trabajo, combinando y movilizando los recursos necesarios para el logro de un resultado excelente y que es validado en una situación de trabajo" (Le Boterf, 200I, citado en MINEDUC, 2007, p. 7).

La OCDE refuerza el hecho de que "una competencia es más que conocimientos y destrezas. Involucra la habilidad de enfrentar demandas complejas, apoyándose en y movilizando recursos psicosociales (incluyendo destrezas y actitudes) en un contexto en particular" (OCDE y DESECO, 2005, p. 3).

Una definición de Competencia TIC o Competencia Digital (CD), acorde a The National Higher Education Information and Communication Technology Initiative, dentro del contexto de una educación superior, es la siguiente:

Competencia TIC es la capacidad de utilizar tecnología digital, herramientas de comunicación, y/o redes apropiadamente para solucionar problemas de información a fin de funcionar en una sociedad de la información. Esto incluye la capacidad de utilizar tecnología como una herramienta para investigar, organizar, evaluar, y comunicar información y la potestad de un entendimiento fundamental de cuestiones éticas/legales en torno al acceso y al uso de la información. (ETS, 2004, citado en Gallardo, Marqués y Gisbert, 201 I, p. 4)

Una definición de Competencia TIC para la Docencia o Competencia Digital Docente (CDD) es la entregada por el MINEDUC:

Las competencias que debe tener un profesor en su práctica y desempeño profesional para mantenerse integrado en la Sociedad del Conocimiento y facilitar que sus estudiantes lo hagan, [se traducen en que] debe apropiarse de un saber hacer con las tecnologías de la información y la comunicación para acompañar procesos pedagógicos, relacionales y 
comunicacionales como de gestión y de desarrollo personal y social. (MINEDUC, 2007, p. 6)

Sin embargo, para efectos de este estudio, una forma interesante de definir tanto la CD como la CDD, donde además se incorporan las creencias, conocimientos y actitudes, enfatizando el concepto de alfabetización múltiple, es la siguiente:

[Competencia digital]... conjunto de valores, creencias, conocimientos, capacidades y actitudes en aspectos tecnológicos, informacionales y comunicativos que nos lleva a una alfabetización múltiple compleja. (Gutiérrez, 20II; Gisbert y Esteve, 20I I; Larraz, 2013, citados en Durán, Gutiérrez y Prendes, 2016, p. 99)

La competencia digital docente reúne todos estos aspectos de la competencia digital, añadiendo además el criterio pedagógico didáctico para la integración efectiva de estos elementos en el proceso de enseñanza-aprendizaje. (Krumsvik, 20II, citado en Durán et al., 2016, p. 99)

La revisión de diversas propuestas en la materia ha permitido conocer los distintos estándares TIC existentes en la literatura, especialmente los de Estados Unidos, Europa y Latinoamérica (Gallardo, Marqués y Gisbert, 20I I). Estos autores destacan los principales estándares existentes: ISTE, QTS, EPICT, UNESCO, ICT Competency Framework (Australia), MACQT - Australia, INSA (Colombia), ENLACES (Chile), cada uno de ellos con enfoques específicos, pero con el fin común de formar o capacitar a los docentes acorde a los nuevos tiempos en las tecnologías digitales.

En Chile se han trabajado estándares para la formación inicial docente. Esta propuesta busca dar respuesta a la necesidad del diseño e implementación de unos estándares TIC que definan un marco consensuado respecto de la preparación de estos profesionales en diversos aspectos relacionados con las tecnologías, tomando en consideración su uso instrumental, curricular y, en general, su impacto en la sociedad (Enlaces y UNESCO, 2008). La propuesta de estándares TIC para la FID considera las siguientes dimensiones: 
Área Pedagógica; Aspectos Sociales, Éticos y Legales; Aspectos Técnicos; Gestión Escolar y Desarrollo Profesional (Enlaces y UNESCO, 2008, p. I45).

¿Qué sucede con estos estándares en el caso de futuros profesores de matemática e informática educativa? ¿Cómo deberían enseñar usando las TIC? ¿Qué recursos deberían utilizar? Existen diversas formas de clasificar los recursos TIC. López (20I I), basándose en el planteamiento de Andee Rubin ${ }^{\mathrm{I}}$, agrupa en cinco categorías los tipos de herramientas para crear ambientes enriquecidos por las tecnologías digitales en los procesos de aprendizaje de las matemáticas: Conexiones dinámicas manipulables (Cabri - Géometrè, Geogebra, Proyecto Descartes, Jclic); Herramientas avanzadas (Mathematica, Maple, Matlab); Comunidades ricas en recursos matemáticos (Iluminations NCTM, National Library of Virtual Manipulatives; Proyecto CICA THALES, DivulgaMAT); Herramientas para explorar la complejidad (Fractint, Simcalc).

En cuanto a la enseñanza y aprendizaje de las matemáticas, las herramientas aisladas no cambiarían significativamente la interacción y los resultados. Lo fundamental es que debe haber una metodología de trabajo adecuada que permita involucrar a los estudiantes - como protagonistas- en el desarrollo de proyectos, la resolución de problemas y la interacción entre pares, en busca de soluciones para el desarrollo del pensamiento crítico y creativo (Oteiza y Miranda, 2003; López, 2011).

Un modelo adecuado para el uso de las TIC en el aula debería recoger ideas y planteamientos, por ejemplo, de la Escuela Nueva, o bien de las teorías del aprendizaje tales como el constructivismo o el socioconstructivismo. Incluso incorporar ideas liberadoras y transformadoras de Paulo Freire, lejos de una educación "bancaria" (Área, 2008). En este modelo, los estudiantes deberían construir el conocimiento a partir del análisis de la información, realizar trabajos colaborativos y, lo fundamental, que el docente debería cambiar su rol

Andee Rubin, científica senior en TERC, ha hecho investigación y desarrollo en los campos de tecnología, investigación educativa y aprendizaje en línea por más de 25 años. https:// www.terc. edu/display/Staff/Andee+Rubin 
al de mediador o supervisor de proyectos más que el de solo trasmisor de conocimientos.

También es posible establecer una relación entre el conocido "triángulo didáctico" y el uso de las TDIC². Quintana (2013) analiza los diferentes tipos de interacciones: sociales, culturales y contextuales, instrumentales y tecnológicas, y finalmente personales. Sin embargo, también existe el lugar que ocupan la TDIC en las interacciones de este modelo triangular (Quintana, 2013). "La actividad mental constructiva del alumno que aprende, la ayuda sostenida y continuada del que enseña, y el contenido que es objeto de enseñanza y aprendizaje" (Onrubia, 2005, citado en Quintana, 2013 , p. 9I). Estas interacciones por lo general son de tipo directo; sin embargo, lo interesante es que pueden además ser mediadas por la TDIC.

Los profesores requieren una formación más sólida en cuanto al conocimiento digital. Por ello es relevante hacer referencia al modelo TPACK de Mishra \& Koehler (2006). Es necesario indagar y profundizar acerca del Conocimiento Tecnológico del Contenido, pero además en el Conocimiento Tecnológico Pedagógico.

"Explora, conjetura, pon a prueba tus ideas,... aprende como seguir”, basado en la idea del Modelo Interactivo para Aprender (Oteiza y Miranda, 2003, p. 7). La experiencia está mostrando que las $\mathrm{TIC}^{3}$ pueden ayudar a proponer cambios en la forma de realizar la clase de matemática, lo que se expresa en cuatro principios: a) centrar la matemática escolar (su aprendizaje, su enseñanza) en el estudiante, b) usar el poder de la visualización ${ }^{4}$, c) fomentar el aprendizaje social y la interacción con la matemática (Vigotsky, P. Freire.), y d) potenciar el aprendizaje de la matemática con el uso de la tecnología.

Tecnologías Digitales de la Información y la Comunicación.

Adaptado de la presentación sobre Modelo Interactivo realizada por Fun Learning Tecnhologies para colegios de la red CIC en la que participó el autor de esta investigación como capacitador y miembro de FLT.

4 Joseph Dalin, http://www.mathematix.com/NovoMath/About_art.htm 


\section{Metodología}

La presente investigación con fines exploratorios y descriptivos, utilizó un enfoque fundamentalmente cualitativo y desde un paradigma esencialmente interpretativo. (González-Monteagudo, 2000).

El tipo de investigación escogida para llevar a cabo los propósitos correspondió al estudio de casos, acorde a los planteamientos de Stake (1998) y Mc Millan y Schumacher (2005). El caso ha sido elegido por su singularidad y para ilustrar un tema específico. El propósito del estudio fue hacer - dentro de los límites de tiempo estipuladosuna descripción detallada del caso y un análisis e interpretación por parte del investigador. Esta investigación utiliza el estudio de caso intrínseco (Rodríguez y Valldeoriola, 2009). El criterio para decidir la elección del caso coincide con el proceso de rediseño curricular que lleva adelante la carrera de PMIE. Particularmente, la mirada está puesta en la componente de Informática Educativa y la formación que han recibido los estudiantes.

Se trabajó una muestra intencionada, la cual tiene que ver con los fines de la investigación (Ruíz, 2003; Canales, 2006). Se impone la profundidad sobre la extensión, explicitando los criterios de elección y saturación (Martínez, 2006; Canales, 2006).

Se utilizó un cuestionario y entrevistas individuales acordes a la disponibilidad de los estudiantes y las del investigador. El cuestionario se confeccionó a partir de la adaptación de instrumentos aplicados en estudios previos. En particular, interesaron los estudios de Bahamondes et al. (20I0), Prendes (2010) y Martínez (20I5). El instrumento además incorporó ítems específicos, considerando que se trata de estudiantes y egresados de Pedagogía en Matemática. El cuestionario propuesto fue sometido a la validación de expertos, en los términos descritos por Canales (2006).

Posteriormente se realizaron entrevistas semiestructuradas con algunos de los sujetos participantes ${ }^{6}$, acorde a la definición de este instrumento entregada por Canales (2006). Esta técnica

\footnotetext{
5 Se pidió autorización formal a la Dra. María Paz Prendes para adaptar su cuestionario a esta investigación.

6 Se entrevistó a dos estudiantes egresados y dos estudiantes de último año.
} 
complementó la aplicación del cuestionario para profundizar en temas claves necesarios y se logró así una visión más completa o integral de las percepciones de los estudiantes. La entrevista fue sometida a la validación de expertos.

En la investigación participaron 24 sujetos (I3 egresados de la PMIE y I I estudiantes de último año). De ellos, I 2 eran damas y 12 eran varones. Del total, 22 estaban en un rango de 20 a 30 años y solo 2 en un rango de 3 I a 40 años. Los egresados (en diciembre de 20I5) recién estaban trabajando en colegios desde marzo de 2016, mientras que los estudiantes de último año estaban haciendo su última práctica profesional y el seminario de grado.

A partir de las preguntas cerradas del cuestionario se realizó primeramente un análisis que incluyó gráficos y tablas de frecuencia. Se utilizó SPSS para un manejo más cómodo de la información cuantitativa. A partir de las respuestas de las entrevistas, se propuso realizar un análisis cualitativo categorial y de contenido (Cáceres, 2003). Básicamente se trabajaron tres etapas: pre-análisis, codificación y categorización. Para facilitar el proceso se utilizó la planilla electrónica Excel®.

\section{Resultados de la investigación}

En relación con las competencias TIC generales, este punto correspondió al Bloque 2A "Aspectos Técnicos" del cuestionario aplicado. En síntesis, se puede concluir que los estudiantes de la PMIE se perciben competentes en general respecto de los aspectos técnicos, es decir, en el conocimiento computacional (respuestas que varían entre 60\% y 92\% según el ítem, al juntar las categorías "normal" y "profundo" o bien al considerar la categoría "Si”") y herramientas más usuales, como procesadores de texto y planillas electrónicas (respuestas que varían entre 51\% y 84\% según el ítem, al juntar las categorías "bastante" y "mucho"), o bien herramientas de búsqueda o comunicación (entre 58\% y 71\% según ítem, al juntar las categorías "bastante" y "mucho"). Sin embargo, las respuestas han mostrado que los encuestados se perciben con menos dominio en herramientas TIC para el trabajo colaborativo (58\%, al juntar las 
categorías "nada” y "poco"), bajo dominio en el uso de los Campus Virtuales (42\% para la categoría "poco"), así como también en las herramientas de publicación (38\% al juntar las categorías "nada” y "poco"), multimedia (34\% al juntar las categorías "nada" y "poco") y uso de lectores RSS (75\% para la categoría "nada”). En el caso de herramientas avanzadas (bases de datos, software matemático de alto nivel y lenguajes de programación), se evidencia que hay sujetos que conocen las herramientas y otros que no las saben usar (entre $46 \%$ y 50\% al juntar las categorías "nada" y "poco").

Respecto del análisis cualitativo, las categorías que aportan a este Bloque 2A son CI (Enseñanza Formal), C3 (Aprendizaje Autónomo), C8 (Desconocimiento de las herramientas digitales). Es decir, los sujetos reconocen, por una parte, una enseñanza formal universitaria que les ha entregado herramientas y software que les permiten desenvolverse en el medio profesional y pedagógico a modo de una base tecnológica. Sin embargo, señalan que hay herramientas o aplicaciones que las han tenido que aprender por su propia cuenta. Por otro lado, existen además recursos que al momento de mencionárselos ellos simplemente nos los conocen. Por ejemplo, lo consultado en el cuestionario sobre uso de lectores RSS o bien sitios y repositorios de recursos digitales específicos ${ }^{7}$.

Efectivamente, la percepción de los estudiantes respecto de sus competencias TIC varía, dependiendo de los recursos involucrados (Arras, et al., 20I I), en este caso herramientas básicas versus avanzadas. O bien, que los futuros docentes están bien capacitados en el uso de tecnología básica, pero no muy familiarizados con tecnologías más avanzadas de la web 2.0 (Lei, 2009). Si bien los sujetos manejan buena parte de las herramientas propuestas, las mayores carencias tienen que ver con la colaboración y gestión de información en grupos (Prendes et al., 2010). Finalmente, los sujetos se perciben competentes en las herramientas básicas de productividad (Bahamondes et al., 2010).

En relación con las competencias TIC para la docencia y la noción de un modelo pedagógico o metodologías apropiadas para el uso de

Illuminations (NCTM), Biblioteca de Manipulativos Virtuales (UTAH), Shodor, Eduteka, DivulgaMat, Proyecto Descartes, Proyecto CICA THALES, entre otros. 
las TIC en el aula, este punto correspondió a los Bloques 2B "Área Pedagógica” y 2C "Gestión Escolar” del cuestionario aplicado. El análisis de las respuestas ha mostrado que los encuestados reconocen criterios adecuados $^{8}$ a la hora de escoger y utilizar recursos TIC en el aula (entre $46 \%$ y $96 \%$ al juntar las categorías "bastante importante" y "muy importante"). No obstante, se perciben con un bajo conocimiento sobre estrategias metodológicas con uso de TIC para la enseñanza y el aprendizaje. Esto se correlaciona con una baja implementación de estas estrategias en el aula y uso de las TIC para evaluar (79\% para la categoría "no usa"). El aspecto metodológico se puede apreciar en los siguientes gráficos.

Gráficos $N^{\circ}$ I y N²: Respuestas a los ítemes 9 y 10.
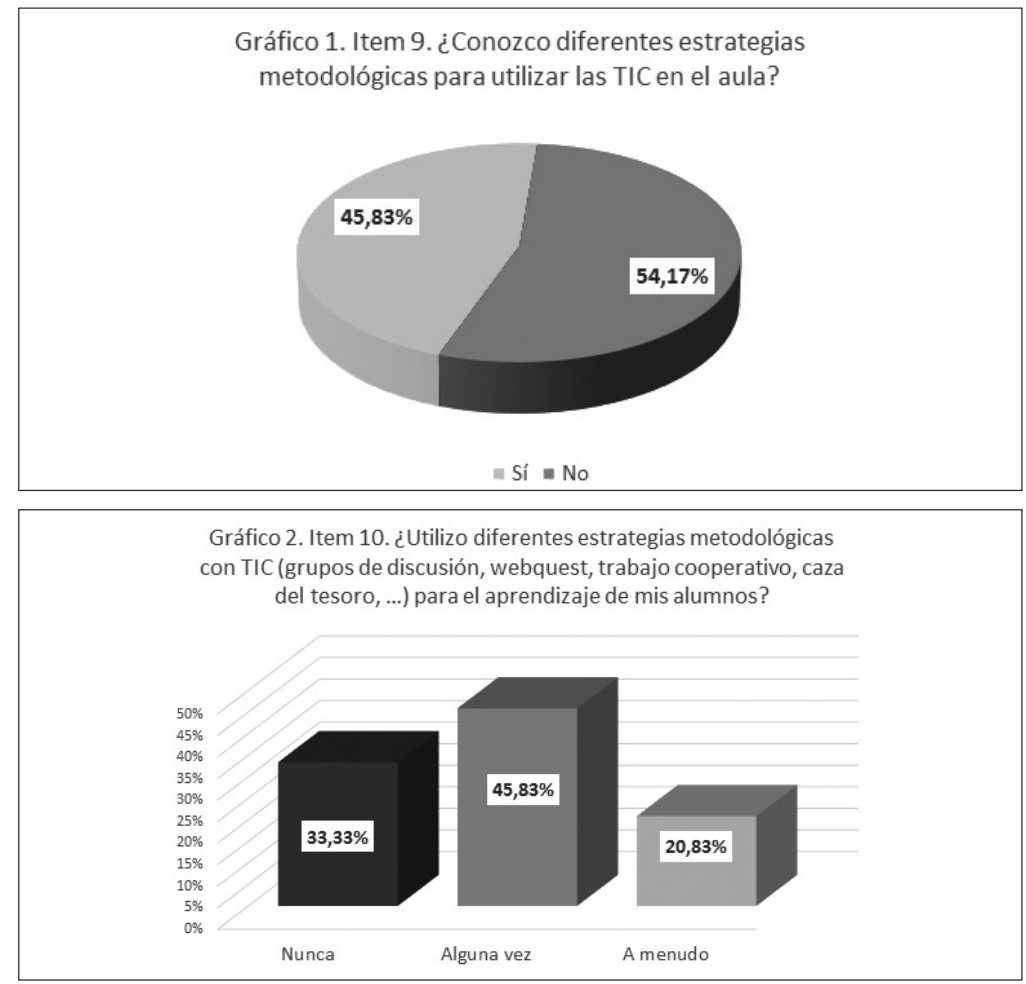

Fuente: SPSS 20.

8 Facilidad de uso, relevancia científica y profesional, innovación tecnológica y disciplinar, resuelve necesidades de aprendizaje, accesibilidad, asequibilidad, tiempo invertido para aprender, recurso motivador. 
El cuestionario ha mostrado que los sujetos expresan un bajo uso de recursos TIC para la comunicación (46\% al juntar las categorías "nada" y "poco") y redes sociales (75\% al juntar las categorías "nada" y "poco") en la gestión docente. Por otra parte, un bajo uso de herramientas TIC para el trabajo colaborativo (75\% para la categoría “nada”) y Campus Virtuales (7I\% al juntar las categorías "nada” y "poco"). Además, una baja motivación o experiencia en la publicación de contenidos educativos en la web (67\% al juntar las categorías "nada" y "poco") y uso de software o herramientas gratuitas (54\% al juntar las categorías "nunca” y "alguna vez"). Finalmente, el uso de herramientas y aplicaciones más avanzadas, como bases de datos, software de alto nivel o la misma programación (entre 58\% y $83 \%$ para la categoría "nada”), quedan más bien fuera del ámbito escolar en su real utilidad para los docentes. Es decir, pueden ser competentes en las herramientas, pero el uso en la gestión es bajo o simplemente no ocurre. Respecto del análisis cualitativo, fundamentalmente a partir de las entrevistas, las categorías que aportan a este punto son C2 (Ausencia de Modelo Pedagógico o metodologías para las TIC en el aula), C4 (Evaluación Tradicional), C5 (Ausencia de cultura de trabajo colaborativo). Algunos ejemplos de la categoría C2 son los siguientes:

S7E3L_109: yo creo que es un tema de las prácticas, porque lo que bablamos, o sea para integrar al aula uno tiene que estar en el aula ..., o sea aprendimos cómo funcionaba el software, sí, sabiamos cómo utilizar la berramienta en sí, cierto, pero el tema de practicarlo ... bacer una clase directamente con el software [metodológico] y acompañado de un público, no, eso no se ve,... lo que babía era solamente ... un estudio en profundidad de estos softwares educativos.

S4E2L_553: Conozco el Office que también sirve, cierto, el Word o también puedo buscar en internet... pero cuando es como aplicar... a la docencia o al enseñar, yo encuentro que abí está... abí está el problema...

S9EIL_25... Entonces claro, por ejemplo, ... yo sé usar Geogebra pero, así como una metodología que me diga cómo usar Geogebra para poder llegar bien al estudiante, no. Yo sé bacer algunas cosas ... pero, así como que me bayan enseñado una didáctica... no... 
Los sujetos reconocen que no se sienten tan preparados para implementar las TIC en el aula, ya que no tienen necesariamente un conocimiento sobre metodologías para utilizar las TIC o bien teorías (modelo) o taxonomías digitales que les den directrices para desenvolverse acorde a una meta más clara. Por ejemplo, sería interesante que conocieran acerca del modelo TPACK y sus implicancias en el conocimiento tecnológico pedagógico. Los sujetos reconocen haber recibido la enseñanza sobre el uso de herramientas digitales o software, pero perciben que no se profundizó tanto en cómo utilizarlas bajo una intencionalidad pedagógica.

Retomando la idea de "modelo pedagógico para el uso las TIC", los sujetos de la PMIE no logran establecer una fuerte relación entre sus asignaturas pedagógicas y/o didácticas, de modo que pudieran aprovechar el conocimiento que ya tienen sobre las teorías clásicas del aprendizaje y actualizaran sus conocimientos a nuevas tendencias, como el conectivismo, por dar un ejemplo. Lo anterior queda reforzado a partir de las respuestas al cuestionario (ítem 27), donde se revela que una amplia mayoría (I8 sujetos, 75\%) desconoce un "modelo pedagógico" o teorías que sustenten la enseñanza con TIC.

S22CML_23: ... en este momento no manejo ningún modelo pedagógico que sustente la enseñanza con TIC.

Se reconoce que las TIC no se usan para evaluar a los alumnos en los colegios, es más habitual usar los métodos tradicionales. Por una parte, esto tiene que ver con el conocimiento de los sujetos acerca de estas herramientas, por ejemplo, los mismos campus virtuales a través de un Moodle, o bien recursos digitales de la eeb que estén orientados o puedan ser utilizados en un esquema evaluativo. Por otro lado, en la práctica se percibe que hay más dificultades para implementar este tipo de innovaciones. A veces, los colegios cuentan con sistemas o plataformas para evaluar "on line" a los estudiantes, pero esto tiene más un carácter institucional que abierto a los docentes. Finalmente, a pesar que los sujetos puedan tener conocimiento sobre herramientas para el trabajo colaborativo (Dropbox, Google Drive,

Oteiza y Miranda (2003); Siemens \& Fonseca (2004); Mishra \& Koehler (2006); Area (2008); Quintana (2013), entre otros. 
etc.), se reconoce que en los colegios no hay una "cultura" justamente de trabajo colaborativo.

Acorde a los estudios previos es posible establecer una cierta correspondencia con estos hallazgos. En general, los futuros docentes “... tienen un nivel aceptable en las habilidades digitales básicas, pero no disponen de un nivel adecuado en la aplicación didáctica de las TIC, y en las estrategias digitales necesarias para su propio desarrollo profesional" (Esteve, 2015, p. 27). Al considerar el estudio que realiza Martínez (2015), los futuros docentes de educación básica tienen una mejor percepción sobre el conocimiento de estrategias metodológicas con uso de TIC (PEB 60\% y PMIE 46\%) y utilización de las mismas, respecto de los estudiantes de la PMIE (PEB 89\% y PMIE 67\% al juntar las categorías "alguna vez" y "a menudo").

Respecto de la valoración por aquellas herramientas para la gestión docente y la enseñanza, los sujetos son capaces de reconocer y valorar una lista de recursos. Las herramientas Word, Excel, Power Point y Geogebra son los más reconocidos. Otras herramientas reconocidas con menor frecuencia son: Graficadores, Prezi, Symbolab, Youtube, Google Drive, Dropbox, Cabri, Educa Plus, JClic, Exe-learning, Thatquiz, Aula Virtual, entre otros. Esto es coherente con la categoría CI (enseñanza formal).

En cuanto a competencias TIC en el área de desarrollo profesional, el análisis de las respuestas del cuestionario (bloque 2D "Desarrollo Profesional") ha mostrado que este punto ha sido uno de los que se percibe más bajo en cuanto a motivación, conocimiento y participación. Por ejemplo, en proyectos de innovación (79\% para la categoría "No"), actividades formativas con TIC (67\% para la categoría "No"), publicación de contenidos de libre acceso (58\% para la categoría "nunca"), participación en foros de reflexión (54\% para la categoría "nunca”) y redes profesionales (60\% para la categoría "nunca”) o grupos de investigación (71\% para la categoría "nunca"). Finalmente, se evidencia poco acceso a repositorios de recursos digitales en la web como los ya mencionados.

En relación al análisis cualitativo, esto se corresponde con la categoría C6 "Falta de incentivo o capacidad para la investigación 
y/o reflexión”. Esto tiene que ver con una percepción de los sujetos entrevistados de que no hay un incentivo real desde la universidad a la investigación en pregrado (salvo el trabajo de tesis en el último año como requisito de egreso). Pero también es el caso de que no hay capacidad o interés personal de hacerlo, ya que los estudiantes por lo general tienen una serie de deberes académicos que tienen que cumplir en forma prioritaria y -en palabras de ellos mismospoco tiempo les queda para otras instancias como proyectos TIC, congresos, foros reflexivos, etc.

Respecto de la planificación de actividades con TIC para apoyar el aprendizaje, la gran mayoría declara haberlo hecho (96\%, para la categoría "Sí"). Es relevante saber que la mayoría de los estudiantes y egresados de la PMIE han planificado actividades escolares con uso de TIC. Al menos lo han realizado en sus prácticas profesionales, donde se les motiva a que lo hagan dependiendo de los recursos disponibles en cada establecimiento.

Finalmente, frente a la pregunta: ¿cómo describiría su dominio (nulo, básico, medio o avanzado) en el uso de TIC para integrar estas tecnologías a sus tareas docentes, tanto en el área pedagógica como en la gestión escolar?, sus respuestas tienden hacia la percepción de un dominio básico a medio (33\% "básico", 59\% “medio" y 8\% "avanzado"). Esto se corresponde con las categorías del análisis cualitativo C2 (Ausencia de Modelo Pedagógico o metodologías para las TIC en el aula), C3 (Aprendizaje Autónomo), C7 (Más matemática que informática educativa), C9 (Pertinencia o no pertinencia de la Malla Curricular) y CIO (Variabilidad de la enseñanza formal recibida).

\section{Síntesis y conclusiones}

A partir de los resultados de la investigación se revela que los estudiantes encuestados de último año y egresados de la PMIE han logrado un desarrollo aceptable en competencias TIC generales, a partir de sus estudios formales en la universidad y también de manera autónoma. Es decir, poseen un repertorio de conocimientos y habilidades que les permiten desenvolverse adecuadamente 
en los "Aspectos Técnicos". No obstante, las diferencias entre los encuestados o falencias podrían explicarse desde el análisis cualitativo y las categorías CI (Enseñanza Formal), C3 (Aprendizaje Autónomo), CI0 (Variabilidad de la enseñanza formal recibida), CII (Motivación personal por usar las TIC), entre otras.

Este mismo conocimiento "técnico" les permitiría un cierto desenvolvimiento en la "Gestión Escolar"; sin embargo, en la práctica esta área reporta algunas falencias, acorde a cómo se desarrollan las cosas en los establecimientos. Es decir, los sujetos pueden dominar las herramientas, pero no necesariamente las ponen en práctica. Por ejemplo, ya se ha reportado un bajo uso en la gestión de las herramientas TIC en aspectos colaborativos o informacionales. Esta situación puede atribuirse en parte a la "cultura escolar", tal como revelan los mismos sujetos en las entrevistas, ya que las herramientas web 2.0 no tienen una funcionalidad explícita o institucionalizada para mejorar los procesos.

Una dimensión que requiere atención es el "Área Pedagógica”, donde los sujetos perciben que desconocen metodologías o un modelo que les permita una mejor integración de las TIC en el aula, acorde a los procesos de enseñanza y aprendizaje de los estudiantes en matemática. Estas falencias podrían explicarse desde las categorías CI (Enseñanza Formal), C2 (Ausencia de Modelo Pedagógico o metodologías para las TIC en el aula), C3 (Aprendizaje Autónomo), C4 (Evaluación Tradicional), C8 (Desconocimiento de las herramientas digitales), CIO (Variabilidad de la enseñanza formal recibida), CI I (Motivación personal por usar las TIC), entre otras. Particularmente, la categoría CIO explicaría que el grupo de sujetos investigados realizó su aprendizaje en los módulos de informática educativa con diferentes profesores, diferentes enfoques y énfasis. Sin embargo, la categoría CI I revela además que hay una cuota personal en el uso o no uso de las TIC.

Otro punto que requiere ser revisado corresponde a la dimensión "Desarrollo Profesional". Al menos en este grupo de estudiantes se revela que todo lo relacionado con investigación en el área, publicación, reflexión y redes de colaboración, etc., no es el fuerte y más bien ha tenido que ver con la motivación personal (CII) y oportunidades en la universidad. En general, esta situación podría 
explicarse mediante la categoría C6 (Falta de incentivo o capacidad para la Investigación y/o reflexión) y cada sujeto vivió un proceso diferente, por lo que la categoría CI I (Motivación personal por usar las TIC) también puede reforzar la comprensión de las falencias en esta dimensión de "Desarrollo Profesional".

Acorde a los resultados de la investigación y en el contexto de una sociedad digital, es recomendable revisar permanente la línea de Informática Educativa de la Pedagogía en Matemática e Informática Educativa de la UCSH, atendiendo fundamentalmente a los aspectos metodológicos del uso de las TIC y la forma de integrarlas efectivamente en el aula.

Si bien la nueva propuesta, en el contexto del rediseño curricular de la carrera, va en la dirección correcta y es un tremendo avance, será necesario seguir trabajando y ajustando los objetivos o competencias de la PMIE para lograr un trabajo más integrado entre las líneas disciplinar, pedagógica e informática educativa, de manera de fortalecer el perfil de egreso de estos futuros docentes. Por ejemplo, se hace imprescindible considerar un trabajo programado de "Simulación de clases con TIC" antes de que los futuros docentes lleguen a trabajar en el aula. Finalmente, ya en las prácticas profesionales, los estudiantes deberían tener instancias de reflexión en torno al uso pedagógico de las TIC. Será importante una participación activa en congresos, eventos y proyectos de innovación.

\section{Referencias bibliográficas}

Area, M. (2008). La innovación pedagógica con TIC y el desarrollo de las competencias informacionales y digitales. Investigación en la Escuela, 64, 5-I8.

Arras, A., Torres, C. y García-Valcárcel, A. (201 I). Competencias en Tecnologías de Información y Comunicación (TIC) de los estudiantes universitarios. Revista Latina de Comunicación Social, 66, I30-III. DOI: I0.4I85/RLCS-66-20I I-927-I30-III. Recuperado el I0 de marzo de 2016 desde http://www.revistalatinacs.org/II/art/927_Mexico/ RLCS_art927.pdf

Bahamondes, V., Aguilar, C. y Torrealba, R. (2010). Competencias IIC en la formación inicial docente: Diagnóstico para el mejoramiento de desempeños profesionales (Informe Final). Santiago: Universidad Católica Silva Henríquez. 
Cabero, J. y Llorente, M. (2006). Capacidades Tecnológicas de las TICs por los estudiantes. Enseñanza, 24, I59-I75.

Cáceres, P. (2003). Análisis cualitativo de contenido: Una alternativa metodológica alcanzable. Revista de la escuela de piscología, 2, 53-82.

Canales, M. (2006). Metodologías de la investigación social. Introducción a los oficios. Santiago, Chile: Editorial LOM.

Durán, M., Gutiérrez, I. y Prendes, M. P. (2016). Análisis conceptual de modelos de competencia digital del profesorado universitario. RELATEC: Revista Latinoamericana de Tecnología Educativa, I5(I), 97-II4. DOI: I0.17398/I695288X.I5.I.97

Esteve, F. (2015). La competencia digital docente. Análisis de la autopercepción y evaluación del desempeño de los estudiantes universitarios de educación por medio de un entorno $3 D$ (tesis doctoral). Universitat Rovira i Virgili.

Enlaces [Enlaces Mineduc]. (2009, diciembre II). Charla Marc Prensky Expo Enlaces 2009 - Parte I [Archivo de video]. Recuperado de https:// www.youtube.com/watch? $v=2$ zwhxmtMLaQ

Enlaces. (2013). Desarrollo de las babilidades digitales para el siglo XXI: ¿Qué dice el SIMCE TIC? Santiago, Chile: LOM. Recuperado el I4 de diciembre de 2015 desde http://www.ceppe.cl/images/stories/recursos/libros_y_cap/ LibroSIMCETICbaja.pdf

Enlaces y UNESCO. (2008). Estándares TIC para la formación inicial docente. Una propuesta en el contexto chileno. Santiago, Chile: LOM.

Gallardo, E., Marqués, L. y Gisbert, M. (20I I). Propuesta de Competencias TIC en el marco del Programa Nacional de Formación y Capacitación Docente (PRONAFCAP). Revista Iberoamericana de Educación, 54(6), I-I3. Recuperado el II de enero de 2016 desde: http://dialnet.unirioja.es / servlet/articulo?codigo $=3698308$

González-Monteagudo, J. (2000). El paradigma interpretativo en la investigación social y educativa: nuevas respuestas para viejos interrogantes. Cuestiones pedagógicas: Revista de ciencias de la educación, I5, 227-246.

Gutiérrez, M., Palacios, A. y Torrego, L. (2010). La formación delos futuros maestros y la integración de las TIC en la educación: anatomía de un desencuentro. Revista de Educación, 352.

Lei, J. (2009). Digital natives as preservice teachers: What technology preparation is needed? Journal of Computing in Teacher Education, 3(85), 87-97.

López, C. (20II). Mejores Prácticas en la Enseñanza de las Matemáticas: La integración de las TICs. SCOPEO. El observatorio de la Formación en Red, 
34. Recuperado el I5 de enero de 2016 desde http://scopeo.usal.es/ enfoque-bol-34-mejores-practicas-en-la-ensenanza-de-las-matematicasla-integracion-de-las-tics/

Martínez, M. (2006). La investigación cualitativa. Síntesis conceptual. Revista IIPSI. Facultad de Psicología. UNMSM, 9(I), I23-I46.

Martínez, V. (2015). Percepciones de Competencias TIC en Formación Inicial Docente de Profesores de Educación Básica. CIAIQ, 5. Recuperado el II de febrero de 2016 desde http:/ / proceedings.ciaiq.org/index.php/ ciaiq2015/article/view/I95/I9I

Matus, C. (2013). Licenciatura en Educación en Física y Matemáticas. Línea de formación intermedia en el uso de tecnologías digitales. Santiago: USACH.

Mc Millan, J. y Schumacher, S. (2005). Investigación educativa. Madrid, España: Pearson.

MINEDUC. (2007). Competencias TIC en la profesión docente. Santiago, Chile: Enlaces/Ministerio de Educación.

Mishra P. \& Koehler M. J. (2006). Technological Pedagogical Content Knowledge: A new framework for teacher knowledge. Teachers College Record, I08(6), I017-1054.

National Council of Teachers of Mathematics, NCTM. (2000). Principles and standards for school mathematics. Reston, VA: NCTM.

OCDE y DESECO. (2005). La definición y selección de competencias clave. Resumen ejecutivo. En Competencias clave en Extremadura, Consejería de Educación y Empleo. Recuperado el I5 de marzo de 2016 desde http:// comclave.educarex.es//130/mod_resource/content/3/DESECO.pdf

Oteiza, F. y Miranda H. (2003). Modelo Interactivo para el Aprendizaje. Proyecto FONDEF D00I1073: "Aprender Matemática Creando Soluciones", Centro Comenius USACH. Santiago, Chile: Zig-Zag.

Ortiz, A., Peñaherrera, M. y Ortega, J. (2012). Percepciones de profesores y estudiantes sobre las TIC. Un estudio de caso. Edutec. Revista electrónica de tecnología educativa, 4I. Recuperado el I8 de diciembre de 2015 desde http://www.edutec.es/revista/index.php/edutec-e/article/ view/352/92

Pedró, F. (2006). The new millennium learners: Challenging our views on ICT and learning. Inter-American Development Bank, 9228.

Perrenoud, P. (2008). Construir competencias desde la escuela. Santiago, Chile: JCSáez.

Prendes, M. P. (2010). Competencias TIC para la docencia en la Universidad Pública Española: Indicadores y propuestas para la definición de buenas 
prácticas: Programa de Estudio y Análisis (Informe de Proyecto No. EA2009-0133). Universidad de Murcia. Recuperado de http:// www. um. es/competenciastic/informe_final_competencias20I0. pdf.

Prendes, M. P., Castañeda, L., y Gutiérrez, I. (2010). Competencias para el uso de TIC de los futuros maestros. Comunicar, Revista Científica De Educomunicación, I8(35). Recuperado el I8 de marzo de 2016 desde: http://dialnet. unirioja.es/servlet/articulo?codigo $=3291776$

Quintana, J. (20I3). La curación o responsabilidad de contenidos, el profesorado como organizador del proceso de aprendizaje mediante Webquests. En J. Bergmann y N. Grané (coords.), La Universidad en la Nube, (pp. 89-I04). Barcelona: Colección Transmedia XXI.

Rodríguez, D. y Valldeoriola, J. (2009). Metodología de la investigación. Barcelona: UOC. Recuperado el 08 de junio de 2016 desde http://cv.uoc.edu/ continguts/PID_O0I48557/index.html

Ruíz, J. (2003). Metodología de la investigación cualitativa. $3^{\text {era }}$ edición. España: Universidad de Deusto Bilbao, Serie Ciencias Sociales.

Siemens, G. (2004). Conectivismo: Una teoría de aprendizaje para la era digital. Recuperado el 4 de febrero de 2016, desde http://www.fce.ues.edu. sv/uploads/pdf/siemens-2004-conectivismo.pdf

Stake, R. (1998). Investigación con estudio de casos. Madrid, España: Morata.

Torres, M. (201 I). Alfabetización digital y actitudes bacia las Tic (tesis doctoral). Universidad Autónoma de Barcelona. Facultad de Ciencias de la Educación. Recuperado el II de enero de 2016 desde https:// www.dropbox.com/s/cro6sxqv8uIa3wn/TESIS\%20FINAL_ POSGRADO.pdf?dl=0

Torres, C. y Arras, A. (20II). Percepción de estudiantes de educación superior sobre sus competencias en las TIC en las universidades Autónoma de Chihuahua y Veracruzana. Apertura, 2(3), I-29.

UNESCO. (2005). Hacia las sociedades del conocimiento. Mayenne, Francia: Unesco. 